\title{
Continuous production of minute virus of mice by an untransformed variant of Fisher rat fibroblast (FR3T3)
}

\author{
Catherine Elaine Koering, ${ }^{1 *}$ Maurice Geuskens ${ }^{2}$ and Jean Rommelaere ${ }^{1,3}$ \\ ${ }^{1}$ Unité d'Oncologie Moléculaire, Institut Pasteur de Lille, Centre National de la Recherche Scientifique URA 1160, \\ BP 245, 59019 Lille Cedex, France, ${ }^{2}$ Département de Biologie Moléculaire, Université Libre de Bruxelles, \\ rue des Chevaux 67, B-1640 Rhode St-Genese, Belgium and ${ }^{3}$ Deutsches Krebsforschungszentrum, \\ Abteilung 0610 and Unité INSERM 375, Im Neuenheimer Feld 242, D-69120 Heidelberg, Germany
}

Many tumour cells are killed by the lytic replication of the autonomous parvoviruses $\mathrm{H}-1$ and minute virus of mice (MVMp), whereas most untransformed cells (although they take up these viruses efficiently) are resistant, i.e. they do not produce infectious virus and are not lysed. Therefore, cells able to continuously produce large quantities of infectious virus have not yet been described. We have isolated such cells from the resistant cell line FR3T3 (Fisher rat fibroblast). These cells (called FR3T3C) produce infectious MVMp virions without being detectably lysed. Furthermore, a per- sistently infected population (R100FR3T3C) was generated by repetitive infection of FR3T3C cells with MVMp. Indeed, R100FR3T3C cells were successfully cultivated for two years and continuously produced infectious virus. Seventeen clones of R100FR3T3C cells isolated by limiting dilution produced infectious virions, indicating that in the R100FR3T3C cell population, virus production was not limited to a few cells. These cell lines may be useful for the production of MVMp and for the generation of a cell line for the packaging of recombinant viral genomes.

\section{Introduction}

The autonomous parvoviruses $\mathrm{H}-\mathrm{l}$ and minute virus of mice (MVMp) are small single-stranded DNA viruses which infect a wide variety of animal species, including humans for H-1 (Siegl, 1984). These viruses are very dependent for their replication on cellular factors which are more specific to transformed cells (Cotmore, 1990; Tattersall \& Gardiner, 1990); their replication is lytic and kills the cell (Tattersall \& Cotmore, 1990; Rommelaere \& Cornelis, 1991). Indeed, the killing by the parvoviruses $\mathrm{H}-1$ or MVMp of a number of human or rodent fibroblasts and epithelial cells increases dramatically when they are transformed in vitro by known oncogenes, chemicals or physical agents (Cornelis et al., 1988a; Mousset et al., 1986; Salome et al., 1990; Van Hille et al., 1989). Cell killing has been dissociated from the lytic production of viral particles, suggesting that specific parvoviral products are cytotoxic (Guetta et al., 1990). This cytotoxicity has been shown to be due to the major non-structural protein, NS-1, and is oncogene-

\footnotetext{
* Author for correspondence. Present address: Laboratoire de Biologie Moléculaire et Cellulaire-Ecole Normale Supérieure de Lyon UMR 49 CNRS-ENS, 46 allée d'Italie, 69364 Lyon Cédex 07, France. $\mathrm{Fax}+3372728080$.

e-mail Catherine.Koering@cri.ens-lyon.fr
}

specific (Brandenburger et al., 1990; Caillet-Fauquet et al., 1990; Cornelis et al., 1988b; Salome et al., 1989, 1990; Van Hille et al., 1989). Conversely, most untransformed cells, although they take up virus efficiently, do not produce infectious virus and are not lysed (Cornelis et al., 1988a 1990; Mousset et al., 1986). Cells able to continuously produce large quantities of infectious virus are therefore difficult to isolate.

Here we report on the characterization of FR3T3C cells, which produce MVMp infectious particles without being lysed. These cells emerged spontaneously from the untransformed FR3T3 (Fisher rat fibroblast) cell line (Salome et al., 1990; Van Hille et al., 1989). When repeatedly infected with MVMp, FR3T3C cells gave rise to a persistently infected cell population (R100FR3T3C) which was successfully cultivated for two years and continuously produced infectious virus.

\section{Methods}

Cells. All cells (see below) were cultured in Dulbecco's modified Eagle's medium (DMEM) supplemented with 5\% fetal calf serum (FCS; Gibco), $1 \%$ glutamine and $12 \mu \mathrm{g} / \mathrm{ml}$ gentamicin. FR3T3 (Fisher rat fibroblast; Salome et al., 1990; Van Hille et al., 1989), FREJ4 cells [FR3T3 cells transformed with the plasmid pSVEJ carrying the $\mathrm{c}-\mathrm{Ha}$ ras gene mutated at codon 12 (Van Hille et al., 1989)], FR3T3C cells (variant of FR3T3), NRK cells (Salome et al., 1989), NIH3T3 cells and A9 cells (variant of mouse $L$ cells) were used in the experiments. 


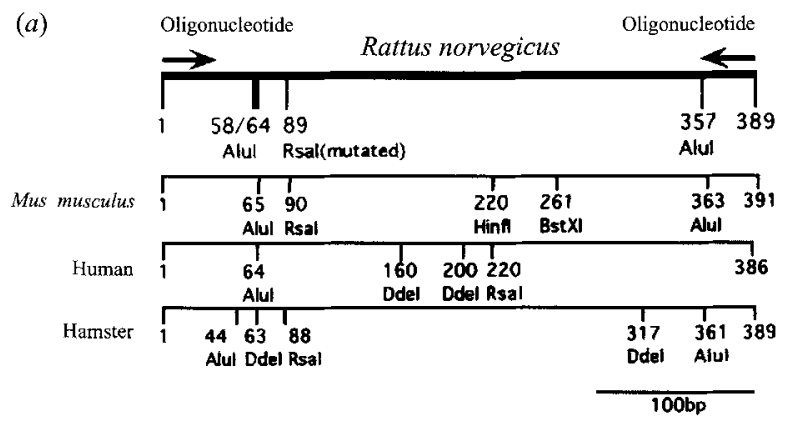

(b)
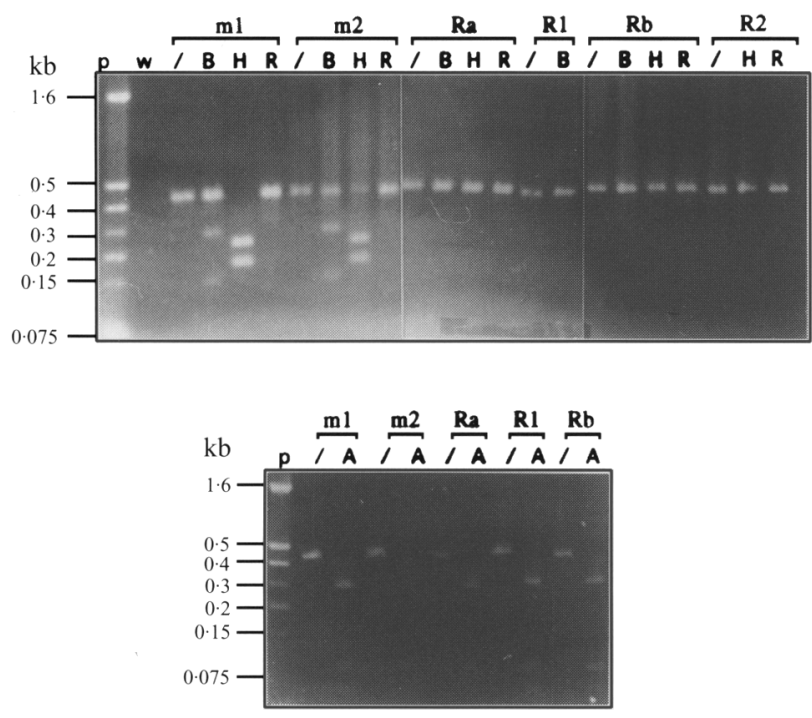

Fig. 1. As shown by RFLP, FR3T3C cells are rat fibroblasts. (a) A mitochondrial DNA fragment (RNA 125 gene) was amplified by PCR from genomic DNA samples isolated from different animal species, using the oligonucleotides located at positions shown by arbitrary numbers and arrows. (b) The PCR-amplified DNA fragments were digested with appropriate restriction enzymes as indicated in $(a)$. The digestion products were separated by electrophoresis in agarose gels and visualized by ethidium bromide staining. Lane p, size marker; lane $\mathrm{w}$, water; lanes $\mathrm{m} 1$, NIH3T3 cells; lanes $\mathrm{m} 2$, ras-transformed NIH3T3 cells; lanes Ra, FR3T3C cells (passage 17); lanes R1, FREJ4 cells; lanes Rb, FR3T3C cells (passage 50); lane R2, FR3T3 cells. Slashes indicate lanes containing non-digested DNA. Restriction enzyme symbols: B, BstXI; H, HinfT; R, RsaI and A, AluI.

\section{Identification of FR3T3C cells}

(i) Karyotypes. Semi-confluent monolayers of A9, FR3T3C, R100FR3T3C and FR3T3 cells were growth arrested with colcemide (Gibco BRL) at a final concentration of $0.06 \mu \mathrm{g} / \mathrm{ml}$ of medium for $4 \mathrm{~h}$. Cells were treated and processed for $\mathrm{R}$ banding (RHG) as described (Dutrillaux \& Couturier, 1981). Chromosomal analysis was done on photographed metaphases. Five to ten spreads were analysed per cell line and 25 cells were examined for total chromosome number in each spread.

(ii) Restriction fragment length polymorphism (RFLP). Extraction of total genomic DNA from NIH3T3, FREJ4, FR3T3 and FR3T3C cells was performed by using standard protocols. The PCR reactions were carried out with approximatively $1 \mu \mathrm{g}$ of total genomic DNA in a final

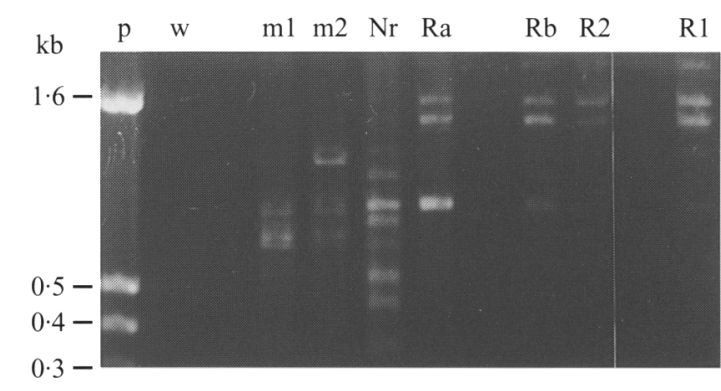

Fig. 2. FR3T3C cells are FR3T3 cell derivatives as shown by RAPD. Genomic DNA isolated from different cell lines was amplified by PCR using the random DNA primer (see Methods). The PCR-amplified DNA fragments were size separated by electrophoresis in agarose gel and visualized by ethidium bromide staining. Lane $\mathrm{p}$, size markers; lane $w$, water; lane $\mathrm{m} 1, \mathrm{NIH} 3 \mathrm{~T} 3$ cells; lane $\mathrm{m} 2$, ras-transformed NIH3T3 cells; lane Nr, NRK (newborn rat kidney) cells; lane Ra, FR3T3C cells (passage 17); lane R2, FR3T3 cells; lane Rb, FR3T3C cells (passage 50); lane R1, FREJ4 cells.

volume of $50 \mu 1$ as previously described (Hänni et al., 1995). The primers used to amplify the $12 \mathrm{~S}$ rRNA mitochondrial genes and the restriction map of the 12S rRNA genes are shown in Fig. 1(a).

The PCR products were digested with the following restriction enzymes: BstXI, DdeI, HinfI, Rsal and AluI, the DNA fragments generated were separated on a $2 \%$ agarose gel (Fig. $1 b$ ).

(iii) Random amplification polymorphism DNA (RAPD). The PCR protocols used in this experiment were adapted from the published RAPD protocols (Hadrys et al., 1992; Welsh et al., 1991). The PCRs were carried out in a total volume of $25 \mu 1$ containing: $10 \mathrm{~mm}-$ Tris-HCl, $0.1 \%$ Triton X-100, $1.5 \mathrm{~mm}-\mathrm{MgCl}_{2}, 0.2 \mathrm{mg} / \mathrm{ml} \mathrm{BSA}, 100 \mu \mathrm{M}-$ dNTPs, $0.4 \mu \mathrm{M}$-primer, $50 \mathrm{ng}$ of cellular DNA and $0.5 \mathrm{U}$ Taq DNA polymerase (Appligene). The primer (5' AACTGACCAACCTGTG 3') was annealed to the genomic DNA at $42{ }^{\circ} \mathrm{C}$ for $1 \mathrm{~min}$ and then 40 cycles of PCR were performed as follows: elongation for $2 \mathrm{~min}$ at $72{ }^{\circ} \mathrm{C}$, denaturation for 1 min at $92^{\circ} \mathrm{C}$. Twenty $\mu$ l of the PCR products was loaded on a $2 \%$ agarose gel (Fig. 2).

MVMp infection and measurement of cell survival after MVMp infection. MVMp (prototype strain) was propagated in A9 cells and purified according to Tattersall et al. (1976). MVMp was titrated by plaque assays on A9 indicator cells (Tattersall et al., 1976). Exponentially growing cultures $\left(5 \times 10^{5}\right.$ cells per $60 \mathrm{~mm}$ dish $)$ were infected with MVMp at various m.o.i.s (1, 10 and 100 p.f.u. per cell) (Koering et al., 1994). Cells were trypsinized $4 \mathrm{~h}$ after mock infection or infection with MVMp and replated at low densities ( 250 to 500 cells) onto $60 \mathrm{~mm}$ dishes. Cell survival was determined by comparing the ability of MVMp-infected and mock-infected cells to form colonies on plastic. Colonies were fixed and stained 5-7 days post-infection (p.i.) as described by Koering et al. (1994).

Measurement of MVMp DNA amplification. The amount of total intracellular viral DNA was measured in whole cell lysates by dispersed cell assay (DCA) (Salome et al., 1989). Cultures $\left(5 \times 10^{5}\right.$ cells per $60 \mathrm{~mm}$ dish) were infected with MVMp at various m.o.i.s, incubated for $2 \mathrm{~h}$ and $30 \mathrm{~h}$, respectively, harvested, and trapped on BA85 nitrocellulose membranes (Schleicher \& Schuell). BA85 filters were processed as described by Salome et al. (1989). As a probe for MVMp, a $5 \mathrm{~kb}$ BamHI fragment excised from pMM984 (Merchlinsky et al., 1983) was radiolabelled with $\left[\alpha_{-}^{32} \mathrm{P}\right] \mathrm{dCTP}$ using the Megaprime DNA labelling system (Amersham). Hybridized radiolabelled DNA was quantified by liquid scintillation spectrometry. MVMp DNA amplification was expressed as a ratio of the amount of hybridized radiolabelled DNA at 
Table 1. FR3T3C cells produce infectious MVMp virions

(a) Determination of infectious virus production of infected cultures $(m . o . i .=1)$ at $30 \mathrm{~h}$ p.i. by plaque assay on $\mathrm{A} 9$ indicator cells. Viral titre in p.f.u./ml. (average of five independent experiments, SD less than $20 \%$ ). (b) Comparison of production of infectious virus. MVMp (prototype strain) was propagated in A9 cells and in FR3T3C cells, purified and plaque-titrated by plaque assays on A9 indicator cells (Tattersall et al., 1976). Viral titre in p.f.u./ml. (c) Infectious centers assays. Percentage of infected cells initiating plaque formation (average of four independent experiments in triplicate, SD less than $20 \%$ ).

\begin{tabular}{|c|c|c|c|c|c|c|}
\hline \multirow[b]{2}{*}{ Cells } & \multirow[b]{2}{*}{ Origin } & \multirow{2}{*}{$\begin{array}{l}\text { (a) Production of } \\
\text { infectious virus } \\
30 \text { h.p.i. }\end{array}$} & \multicolumn{2}{|c|}{$\begin{array}{l}\text { (b) Production of infectious } \\
\text { virus } 6 \text { days p.i. }\end{array}$} & \multicolumn{2}{|c|}{ (c) Infectious centres } \\
\hline & & & m.o.i. $5 \times 10^{-3}$ & m.o.i. 10 & m.o.i. 1 & m.o.i. 10 \\
\hline A9 & $\begin{array}{l}\text { Derived } \\
\text { from mouse } \\
\mathrm{L} \text { cells }\end{array}$ & $9.5 \times 10^{9}$ & $1.9 \times 10^{9}$ & ND & $84 \%$ & $68 \%$ \\
\hline FR3T3C & $\begin{array}{l}\text { FR3T3 cell } \\
\text { variant }\end{array}$ & $6.9 \times 10^{6}$ & $10^{4}$ & $2.5 \times 10^{9}$ & $2 \%$ & $67 \%$ \\
\hline R100FR3T3C & $\begin{array}{l}\text { FR3T3C } \\
\text { persistently } \\
\text { infected cell }\end{array}$ & $0.6 \times 10^{6}-1.6 \times 10^{8 *}$ & ND & ND & $21 \%-64 \%$ & $21 \%-64 \%$ \\
\hline
\end{tabular}

* Persistently infected cells. ND, not determined.

$30 \mathrm{~h}$ p.i. compared to that at $2 \mathrm{~h}$ (input) p.i. for the different m.o.i.s $(0 \cdot 1$, 1 and 10).

Production of infectious $M V M p$ virions. A9 cells infected at an m.o.i. of $5 \times 10^{-3}$ and FR3T3C cells infected at an m.o.i. of $5 \times 10^{-3}$ or 10 , were compared quantitatively for their production of infectious virus by the standard method of propagation and purification (Tattersall $e t$ al., 1976). Viral particles produced were titrated by plaque assay on A9 cells (See Table $1 b$ ).

For determination of infectious virus production, infected cultures $(\mathrm{m} .0 . \mathrm{i} .=1)$ were frozen at $30 \mathrm{~h} \mathrm{p}$.i. and subjected to three freeze-thaw cycles. The suspensions were diluted in culture medium without serum and the released particles were titrated by plaque assay on A9 indicator cells (See Table 1 $a$ ).

Production of virions was also evaluated by the infectious centre assay. Appropriate numbers of persistently infected R100FR3T3C and virus-infected (m.o.i. $=1$ or 10) A9 and FR3T3C cells were treated as described by Salome et al. (1989) and the proportion of productively infected cells was determined 6 days later, as the fraction of seeded cells producing plaques (See Table $1 c$ ).

R100FR3T3C cells and clonal populations of R100FR3T3C cells selected by limiting dilution, were analysed for their capacity to produce infectious virus by the modified plaque assay (Faisst et al., 1989). The last wash of R100FR3T3C cells before plating for limiting dilution was added to A9 cells and did not detectably induce cell mortality after 1 week in culture. A9 cultures $\left(3 \times 10^{5}\right.$ cells) were infected with MVMp virus produced by FR3T3C cells or by A9 cells (m.o.i. $=10^{-4}$ or $10^{-2}$ ) with $0.5 \mathrm{ml}$ of filtered (Minisart NML $0.20 \mu \mathrm{m}$ pore size; Sartorius) lysates of $10^{6}$ cells of R100FR3T3C cells and clones. The production of infectious viruses in A9 cells was evaluated $30 \mathrm{~h}$ p.i. by the modified plaque assay on BA85 filters as described by Faisst et al. (1989). As a probe for MVMp, the $5 \mathrm{~kb}$ BamHI fragment excised from pMM984 was radiolabelled with $\left[\alpha{ }^{32} \mathrm{P}\right] \mathrm{dCTP}$. Hybridized radiolabelled DNA was quantified by liquid scintillation spectrometry.

\section{Results and Discussion}

FR3T3C cells emerged spontaneously from a culture of FR3T3 cells (Fisher rat fibroblast) and were phenotypically indistinguishable from FR3T3 cells, i.e. they had a similar morphology, the same doubling time and did not grow in semi-solid medium or as tumours in nude mice (not shown). However, FR3T3C cells differed from FR3T3 cells by their karyotype and their ability to amplify the MVMp genome and to produce infectious virions.

\section{FR3T3C cells have a different karyotype than FR3T3 cells but are Fisher rat fibroblasts}

In order to characterize FR3T3C cells, karyotypes were made. As compared to FR3T3 cells which have 42 chromosomes, FR3T3C cells had an unusual number of chromosomes, which varied from 66 to 74 , the average number being 72 for FR3T3C cells. However, we did not find a single FR3T3C cell with 42 chromosomes (not shown).

In order to ascertain the origin of FR3T3C cells [and to rule out any possibility of cell contamination (NelsonRees \& Flandmeyer, 1977)] we first verified that they were rat cells by RFLP (Hänni et al., 1995) on mitochondrial DNA (12S rRNA). A mitochondrial DNA fragment (12S RNA gene; Fig. $1 a$ ) was amplified by PCR from total DNA isolated from FR3T3C, NIH3T3 (mouse), FR3T3 (rat) and FREJ4 (rat) cells. The PCR-amplified DNAs were digested with the enzymes BstXI, HinfI, RsaI or DdeI which allow discrimination between human, mouse, rat and hamster species. As a control we used the $A l u \mathrm{I}$ enzyme cutting sites. The comparison of the digestion patterns of the PCR-amplified DNA fragments demonstrated that FR3T3C were rat cells [Fig. $1 b$; the $D d e I$ digestion clearly showed that FR3T3C cells are not hamster cells (not shown)].

We next ascertained by RAPD (Hadrys et al., 1992; Welsh et al., 1991) that FR3T3C cells were indeed FR3T3 cell derivatives. We therefore compared the 


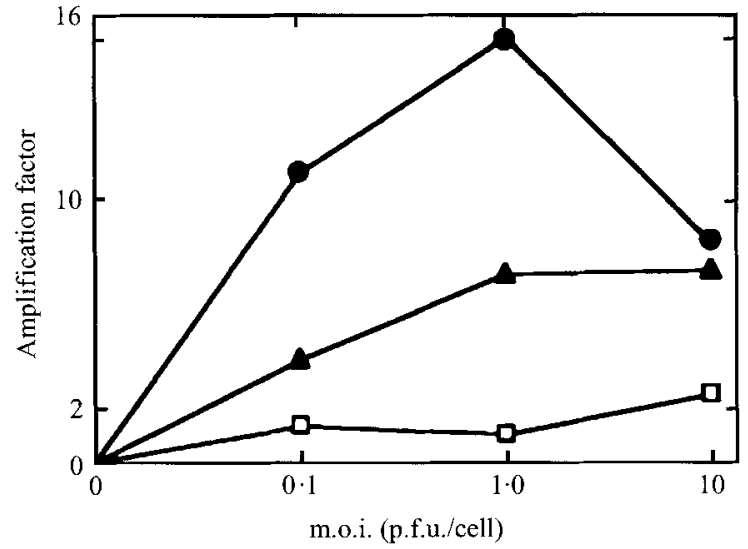

Fig. 3. FR3T3C cells amplify MVMp DNA. FR3T3C cells ( ), A9 cells $(\boldsymbol{A})$ or FR3T3 cells $(\square)$ were infected with MVMp virions at an m.o.i. of $0 \cdot 0,0 \cdot 1,1 \cdot 0$ or $10 \mathrm{p}$.f.u./cell. The MVMp DNA amplification factor was determined as described in Methods.

RAPD patterns obtained with the DNA isolated from FR3T3C cells to the RAPD patterns obtained with the DNA isolated from NIH3T3 mouse cells, NRK, FREJ4 and FR3T3 rat cells used as controls. The RAPD patterns obtained with the DNA of FR3T3C, FR3T3 and FREJ4 cells were similar but different from those obtained with the DNA of NIH3T3 and NRK cells (Fig. 2). These results strongly suggest that FR3T3C cells are FR3T3 cell derivatives.

\section{FR3T3C cells amplify MVMp DNA}

A9 cells, FR3T3 cells and FR3T3C cells were infected at m.o.i.s of $0 \cdot 1,1$ and 10 p.f.u./cell with MVMp virions produced by A 9 cells (see Methods). When the amount of intracellular viral DNA was measured at $2 \mathrm{~h} \mathrm{p}$.i. and $30 \mathrm{~h}$ p.i. in whole cell lysates by DCA, we found that the viral DNA was amplified similarly in FR3T3C cells and in the permissive A9 cells, but was not amplified in FR3T3 cells (Fig. 3). These results indicated that the viral DNA was replicated in FR3T3C cells.

\section{FR 3T3C cells produce infectious MVMp virions}

We next tested the capacity of FR3T3C cells to produce infectious virions 6 days p.i. by plaque assay on A9 cells indicator cultures. The results presented in Table $1(a)$ demonstrate that FR3T3C were able to produce infectious virus. However, FR3T3C cells had to be infected at an m.o.i. of 10 (Table $1 b$ ) as compared to an m.o.i. of $5 \times 10^{-3}$ for A9 cells, to produce at 6 days p.i. an infectious virus titre of $10^{8}-10^{9}$ p.f.u. $/ \mathrm{ml}$. This observation was confirmed by infectious centres assays (Table $1 c)$.

The results described above strongly suggest that the

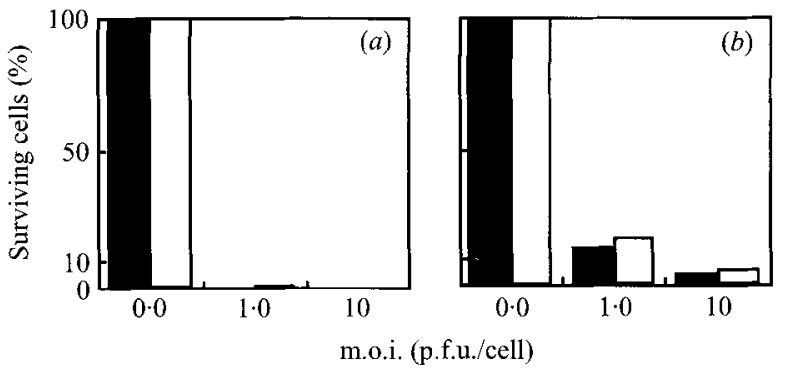

Fig. 4. Transformed cells are infected and killed by MVMp virions produced by FR3T3C cells. A9 mouse cells $(a)$ or FREJ4 rat celts $(b)$ were infected at m.o.i. of $0.0,10$ or 10 with MVMp virions produced either by A9 cells (black bars) or FR3T3C cells (open bars). The percentage of cells surviving infection was determined as described in Methods. ( $a$ and $b$ represent the average of three independent experiments in triplicate, SD less than $15 \%$ ).

rat cells FR3T3C produce MVMp virions able to infect and kill A9 mouse cells. However, it remained to be established whether MVMp virions produced by FR3T3C cells could infect and kill permissive rat cells. To do so, we compared the survival of A9 mouse cells and FREJ4 rat cells [ras-transformed FR3T3 cells highly sensitive to infection by MVMp (Salome et al., 1990; Van Hille et al., 1989)] infected by MVMp virions produced either by A9 cells or FR3T3C cells. The percentage of A9 cells and FREJ4 cells killed by MVMp infection was similar whether the MVMp virions used were produced by FR3T3C cells or by A9 cells (Fig. $4 a$, $b$ ).

\section{R100FR3T3C cells produce MVMp virions continuously}

Since most of the cells producing infectious MVMp virions are killed and cannot be kept in long-lasting cultures, we next evaluated whether FR3T3C cells survived MVMp production and whether MVMpproducing FR3T3C cell lines could be established.

When compared to FR3T3 cells which are resistant to MVMp killing ( $65.4 \%$ of cells survive at day $6 \mathrm{p}$.i. at an m.o.i. of 10 ; Fig. 5) and to A9 cells which are highly sensitive to MVMp killing ( $0 \%$ cells survive at day 6 p.i. at an m.o.i. of 10; Fig. 5) FR3T3C cells were highly resistant to MVMp killing [95.8\% cells survive at day 6 following an infection at an m.o.i. of 10 (Fig. 5) and more than $87 \%$ of the cells survived an infection at an m.o.i. of 100 (data not shown)]. Accordingly, it was possible to establish a cell line of FR3T3C cells (R100FR3T3C) following two subsequent rounds of infection (m.o.i. $=10$ then m.o.i. $=100$ ). This cell line has been cultivated for about two years and still produces infectious MVMp virions (Table $1 a^{*}$ ). Indeed, infection of A9 indicator cells with the filtered supernatants of R100FR3T3C cultures (modified plaque assay) showed 


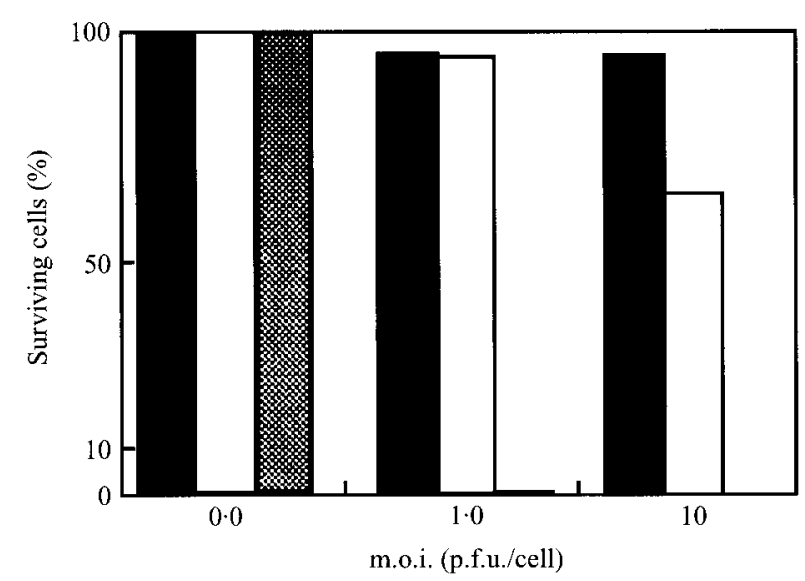

Fig. 5. The production of infectious MVMp virions by FR3T3C cells is not lytic. FR3T3C cells (black bars), FR3T3 cells (open bars), A9 cells (grey bars) were infected with MVMp virions at m.o.i. $0 \cdot 0,1 \cdot 0$ or 10. The percentage of surviving cells was determined as described in Methods (average of 6 independent experiments in triplicates, SD less than $15 \%$ ).

that, independent of the number of passages, infectious MVMp virus was produced by R100FR3T3C cells (not shown). It must be noticed that R100FR3T3C cells did not withstand transformation by the oncogene ras. Indeed, it was not possible to obtain persistently infected clones expressing the $\mathrm{p} 21^{\text {ras }}$ protein from R100FR3T3C cells transfected with pSVEJ (Van Hille et al., 1989) and selected by G418 (data not shown). These last results, in accordance with all previous reports, strongly suggest that R100FR3T3C cells are sensitized to MVMp lytic effect by transformation.

Finally, we evaluated whether individual R100FR3T3C cells isolated by limiting dilution would give rise to clonal populations producing infectious viruses. Seventeen clonal populations were analysed for MVMp production by modified plaque assay on A9 cells. All clones produced infectious virus (not shown). This suggests that in the R100FR3T3C cell line, the production of MVMp virions is not limited to a few cells.

In conclusion, this is the first report on untransformed cells continuously producing infectious parvoviruses. We must stress the fact that FR3T3C could also amplify H1 viral DNA and produce infectious $\mathrm{H}-1$ viruses nonlytically (not shown). At present we do not know why FR3T3C cells produce MVMp virons while the parental untransformed FR3T3 cells do not. Nevertheless, the FR3T3C cells are of high potential interest for the production of large quantities of infectious MVMp and $\mathrm{H}-1$ virions, or to be used as packaging cells lines to generate recombinant parvoviruses.

We thank M. Venin-Fikry and F. Gross for technical assistance and J. Coll, C. Hänni, V. Laudet, A. Mahe and N. Salomé for stimulating and helpful discussions. We are grateful to C. Sutter and P.C.A.
Strangee for critical reading of the manuscript. This work was supported by grants from the Centre National de la Recherche Scientifique, the Institut National de la Recherche Médicale et de la Santé and the Institut Pasteur de Lille, and by fellowships from the Institut Pasteur de Lille and the Fondation des Treilles (to C.E.K.) and by fellowships from Belgian National Fund for Scientific Research (to M.G.).

\section{References}

Brandenburger, A., Legendre, D., Avalosse, B. \& Rommelaere, J. (1990). NS-1 and NS-2 proteins may act synergistically in the cytopathogenicity of parvovirus MVMp. Virology 174, 576-584

Caillet-Fauquet, P., Perros, M., Brandenburger, A., Spegelaere, P. \& Rommelaere, J. (1990). Programmed killing of human cells by means of an inducible clone of parvoviral genes encoding nonstructural proteins. EMBO Journal 9, 2989-2995.

Cornelis, J. J., Becquart, P., Duponchel, N., Salome, N., Avalosse, B. L., Namba, M. \& Rommelaere, J. (1988a). Transformation of human fibroblasts by ionizing radiation, a chemical carcinogen, or simian virus 40 correlates with an increase in susceptibility to the autonomous parvoviruses $\mathrm{H}-1$ virus and minute virus of mice. Journal of Virology 62, 1679-1686.

Cornelis, J. J., Spruyt, N., Spegelaere, P., Guetta, E., Darawshi, T., Cotmore, S. F., Tal, J. \& Rommelaere, J. (1988b). Sensitization of transformed rat fibroblasts to killing by parvovirus MVM correlates with an increase in viral gene expression. Journal of Virology 62, 3438-3444.

Cornelis, J. J., Chen, Y. Q., Spruyt, N., Duponchel, N., Cotmore, S. F., Tattersall, P. \& Rommelaere, J. (1990). Susceptibility of human cells to killing by the parvoviruses $\mathrm{H}-1$ and minute virus of mice correlates with viral transcription. Journal of Virology 64, $2537-2544$.

Cotmore, S. F. (1990). Gene expression in autonomous Parvoviruses. In Handbook of Parvoviruses, vol. I, pp. 141-154. Edited by P. Tijssen. CRC Press: Boca Raton, USA.

Dutrillaux, B. \& Couturier, J. (1981). Technique de laboratoire, pratique de l'analyse chromosomique, pp. 40 45. Paris: Masson.

Faisst, S., Schlehofer, J. R. \& ZUR Hausen, H. (1989). Transformation of human cells by oncogenic viruses supports permissiveness for parvovirus H-1. Journal of Virology 63, $2152-2158$

Guetta, E., Mincberg, M., Mousset, S., Bertinchamps, C., ROMMELAERE, J. \& TAL, J. (1990). The selective killing of transformed rat cells by minute virus of mice does not require infectious virus production. Journal of Virology 64, 458-462.

Hadrys, H., Balick, M. \& Schierwater, B. (1992). Applications of random amplified polymorphic DNA (RAPD) in molecular ecology. Molecular Ecology 1, 55-63.

Hänni, C., Laudet, V., Barriel, V. \& Catzeflis, F. M. (1995). Evolutionary relationships of Acomys and other murids (Rodentia, Mammalia) based on complete 12S rRNA mitochondrial gene sequences. Israel Journal of Zoology 41, 131-146.

Koering, C. E., Dupressoir, T., Plaza, S., Stehelin, D. \& ROMMELAERE, J. (1994). Induced expression of the conditionally cytotoxic herpes simplex virus thymidine kinase by means of a parvoviral regulatory circuit. Human Gene Therapy 5, 457-463.

Merchlinsky, M. J., Tattersall, P., Leary, J. J., Cotmore, S. F., Gardiner, E. M. \& WARD, D. C. (1983). Construction of an infectious molecular clone of the autonomous parvovirus minute virus of mice. Journal of Virology 47, 227-232.

Mousset, S., Cornelis, J. J., Spruyt, N. \& Rommelaere, J. (1986) Transformation of established murine fibroblasts with an activated cellular Harvey ras oncogene or the polyoma virus middle $\mathrm{T}$ gene increases cell permissiveness to parvovirus minute virus of mice. Biochimie 68, 951-956.

Nelson-Rees, W. A. \& Flandermeyer, R. R. (1977). Inter- and intraspecies contamination of human breast tumor cell lines $\mathrm{HBC}$ and BrCa5 and other cell cultures. Science 185, 1343-1344.

Rommelaere, J. \& CoRnelis, J. J. (1991). Antineoplasic activity of parvoviruses. Journal of Virological Methods 33, 233-251. 
Salome, N., Van Hille, B., Duponchel, N., Meneguzzi, G., Cuzin, F., Rommelaere, J. \& Cornelis, J. J. (1990). Sensitization of transformed rat cells to parvovirus MVMp is restricted to specific oncogenes. Oncogene 5, 123-130.

Salome, N., Van Hille, B., Geuskens, M. \& Rommelaere, J. (1989). Partial reversion of conditional transformation correlates with a decrease in the sensitivity of rat cells to killing by the parvovirus minute virus of mice but not in their capacity for virus production: effect of a temperature-sensitive v-src oncogene. Journal of Virology 63, 4797-4807.

SIEGL, G. (1984). Biology and pathogenicity of autonomous parvoviruses. In The Parvoviruses, pp. 297-362. Edited by K. I. Berns. New York: Plenum Press.

TATTERSALl, P. \& COTMORE, S. F. (1990). Reproduction of autonomous parvoviruses. In Handbook of Parvoviruses, vol. I, pp. 123-140. Edited by P. Tijssen. CRC Press: Boca Raton, USA.
Tattersall, P. \& Gardiner, E. M. (1990). Autonomous parvovirushost-cell-interactions. In Handbook of Parvoviruses, vol. I, pp. 111-121. Edited by P. Tijssen. CRC Press: Boca Raton, USA.

Tattersall, P., Cawte, P. J., Shatkin, A. J. \& Ward, D. C. (1976). Three structural polypeptides coded for by minute virus of mice, a parvovirus. Journal of Virology 20, 273-289.

Van Hille, B., Duponchel, N., Salome, N., Spruyt, N., Cotmore, S. F., Tattersall, P., Cornelis, J. J. \& Rommelaere, J. (1989). Limitations to the expression of parvoviral nonstructural proteins may determine the extent of sensitization of EJ-ras-transformed rat cells to minute virus of mice. Virology 171, 89-97.

Welsh, J., Petersen, C. \& McClelland, M. (1991). Polymorphisms generated by arbitrarily primed PCR in the mouse: application to strain identification and genetics. Nucleic Acids Research 19, 303-306.

(Received 8 August 1995; Accepted 5 October 1995) 